\title{
AMIBIASIS INTESTINAL Y EMBARAZO
}

\section{Dr. Rubén Darío Guzmán A.}

Profesor Asociado del Depto. de Obstetricia y Ginecología de la Universidad Nal. de Colombia.

\section{Dr. Jaime Cantillo G.}

Profesor Asistente del Depto. de Anatomía Patológica de la Universidad Nal. de Colombia.

Por todos es bien conocida la alta frecuencia de la amibiasis intestinal humana en todo el mundo especialmente en los países de América Tropical, frecuencia en relación directa con el estado socio-económico, el medio ambiente y el nivel sanitario de la comunidad. Así, es llamativo que en Nueva York y otras ciudades importantes con niveles socio-económico, ambiental y sanitario elevados solamente están infectados del $1 \%$ al $4 \%$ de la población; estas cifras hacen contraste con regiones rurales del sur do los Estados Unidos, en donde estos factores son muy precarios, siendo la cifra de infectados el 30\% (1). Según la doctora Eva Escorcia (2) la frecuencia de la amibiasis intestinal en algunos países y ciudades es la siguiente:

$\begin{array}{lrr}\text { Río de Janeiro } & 3 & \% \\ \text { Saigón } & 5 & \% \\ \text { Santo Domingo } & 6 & \% \\ \text { Estados Unidos } & 10 & \% \\ \text { Egipto } & 8,14 \% \\ \text { Java } & 13 & \% \\ \text { Singapur } & 16 & \% \\ \text { Irak } & 13 & \% \\ \text { Panamá y Somalia } & 45 & \% \\ \text { Hawaii } & 71 & \% \\ \text { Kanerun } & 75 & \% \\ \text { Congo } & 84 & \% \\ \text { Congo } & 84 & \%\end{array}$

Una publicación de William Rojas y cols. (3) dá una incidencia mundial, según diversos autores, que varía entre el $40,3 \%$ al $73 \%$.

En Colombia se han realizado varios trabajos sobre este tema con da-

\begin{tabular}{llrr}
\hline Autor & Lugar & Año & Porcentaje \\
\hline Kofoil (4) & Santa Marta & 1926 & $53,7 \%$ \\
Esguerra (5) & Bogotá & 1937 & $20,1 \%$ \\
Restrepo (6) & Medellín & 1939 & $26,1 \%$ \\
Domínguez (7) & Villavicencio & 1950 & $31,5 \%$ \\
Botero (8) & Medellín & 1958 & $35,3 \%$ \\
Faust E. C. (9) & Cali & 1958 & $40,3 \%$ \\
Duque (10) & Chocó & 1959 & $60 \%$ \\
Restrepo (11) & Amazonas & 1962 & 55 \\
Zuluaga (12) & Santo Domingo (Antioquia) & 1965 & $66 \%$ \\
\hline
\end{tabular}


tos de frecuencia sumamente variables:

Las publicaciones sobre embarazos y amibiasis son muy escasas. Oscar Duque en Antioquia (13) trata sobre tal concomitancia y sus complicaciones fatales. Según Pérez M. L., Gren Way y Nolting D. E. citados por Juan León (14), la amibiasis intestinal se presenta en un alto porcentaje durante el estado grávido-puerperal $(52,9 \%)$, siendo mayor en la gestación que en el puerperio.

Al revisar, con base en exámenes coprológicos, las mujeres embarazadas de la Consulta Prenatal de la Clínica San Pedro Claver del ICSS. encontramos una incidencia del $26 \%$, cifra que podría elevarse un tanto si se tiene en cuenta que ese procedimiento solo se efectúa una sola vez y apenas es un medio ocasional para hacer el diagnóstico de tal afección. En el Centro de Higiene No 9 del Distrito Especial de Bogotá la incidencia es del 34\%; en el Instituto Materno Infantil de Bogotá de 38\%; estas cifras que pueden ser mayores teniendo presente lo arriba anotado.

Las formas de presentarse no difieren de las de la mujer no gestante. Así, en la forma aguda (disentería amibiana) existe sensación de tenesmo anal, deposiciones mucosas o sanguinolentas; dolor abdominal sobre todo en la región del segmento distal del íleon, ciego, apéndice, coIon ascendente 0 a nivel del asa sigmoide y recto; pueden existir además fiebre, vómito y agotamiento marcado. Diferentes opiniones existen sobre la influencia que la amibiasis intestinal ejerce sobre el feto. Pérez M. L. (15) sostiene que en la embarazada la forma aguda no se modifica pero la infección prolongada acarrea un peligro vital para la madre y para el feto; !a amibiasis crónica sue- le reagudizarse con la gestación. Clifford y cols. (16) conceptúan que cuando se presenta diarrea con hepatomegalia durante el embarazo debe pensarse en la posibilidad de una amibiasis y por lo tanto tratarse de inmediato. Según Juan León (14), cuando la forma aguda se prolonga, es casi siempre fatal para el feto; en cambio, la forma latente no parece perturbar la evolución del embarazo ni el puerperio.

Hoy en día es aceptado que el es. tado grávido-puerperal puede ofrecer condiciones propicias para las lesio. nes amibianas latentes y reactivarse con el embarazo. Mitra (17) conside. ra que el embarazo aumenta la gra. vedad de la infección. Experimentos de Teodorovic, Ingallas, Greenberg (18), Biagi, Robledo, Servin y Marvan (19), han demostrado que los corticoides y la progesterona, que se producen en cantidad abundante durante el embarazo, agravan la infección amibiana en los animales de experimentación. Duque O. (13) presenta 12 casos fatales de amibiasis intestinal en mujeres grávidas, demuestra que la enfermedad fue particularmente virulenta; algunos casos se acompañaron de perforación intestinal, otros de abscesos hepáti$\cos$ y pulmonares y unos tantos de colitis gangrenosa. Estudios de Santoyo, Villalobos y Vásquez en México (20) presentan 100 casos de abscesos hepático-amibianos; 6 ocurrieron en embarazadas, 3 de las cuales fallecieron a consecuencia de esta parasitosis. Rivera (21) ha señalado la amibiasis como responsable de partos prematuros; Juan León cree que el motivo por el cual la gravidez ejerce una influencia desfavorable sea debida a causas anatómicas, como son la compresión del asa sigmoide y del recto por el útero grávido, la hiperemia venosa, la cons- 
tipación con exaltación de la virulencia de la flora intestinal y la hipovitaminosis. Duque O. (13) se pregunta si el alto nivel hormonal del embarazo no sería el responsable de la exaltación de la virulencia de la amibiasis. También se ha especulado sobre si los corticoides favoracen la invasividad de las amibas, concepto que comparte Mody U. R. (21); sin embargo Villarejos (23) no pudo comprobar tal afirmación en ratas infectadas de amibas, tratadas con cortisona.

\section{Material y Método}

Se presentan 6 historias clínicas en donde indudablemente se demuestra la agresividad de la amibiasis intestinal asociada con el embarazo, manifestada por perforación intestinal, absceso hepático, empiema pulmonar, como pudo comprobarse en las autopsias efectuadas en cada uno de los casos. En ningún caso se hizo un diagnóstico previo y por consiguiente un tratamiento antiamibiano, y los resultados fueron fatales.

Es llamativa la variedad de diagnósticos erróneos que se hicieron, con agravación del cuadro clínico en perjuicio de las enfermas; estas condiciones nos obligan a pensar más en tal entidad asociada al embarazo con sus complicaciones consecuentes, en toda paciente embarazada con episodios frecuentes de diarreas, y aún más con pujo y tenesmo.

\section{PRIMER CASO :}

HISTORIA CLINICA No 33097.

Edad: 30 años.

Fecha de Ingreso: XI 4/64.

Fecha de muerte: XI 11/64.

Causa de Ingreso: Dolor abdominal en bajo vientre $y$ hemorragia genital.

Enfermedad actual: La paciente ingresa al servicio con un embarazo de aproximadamente

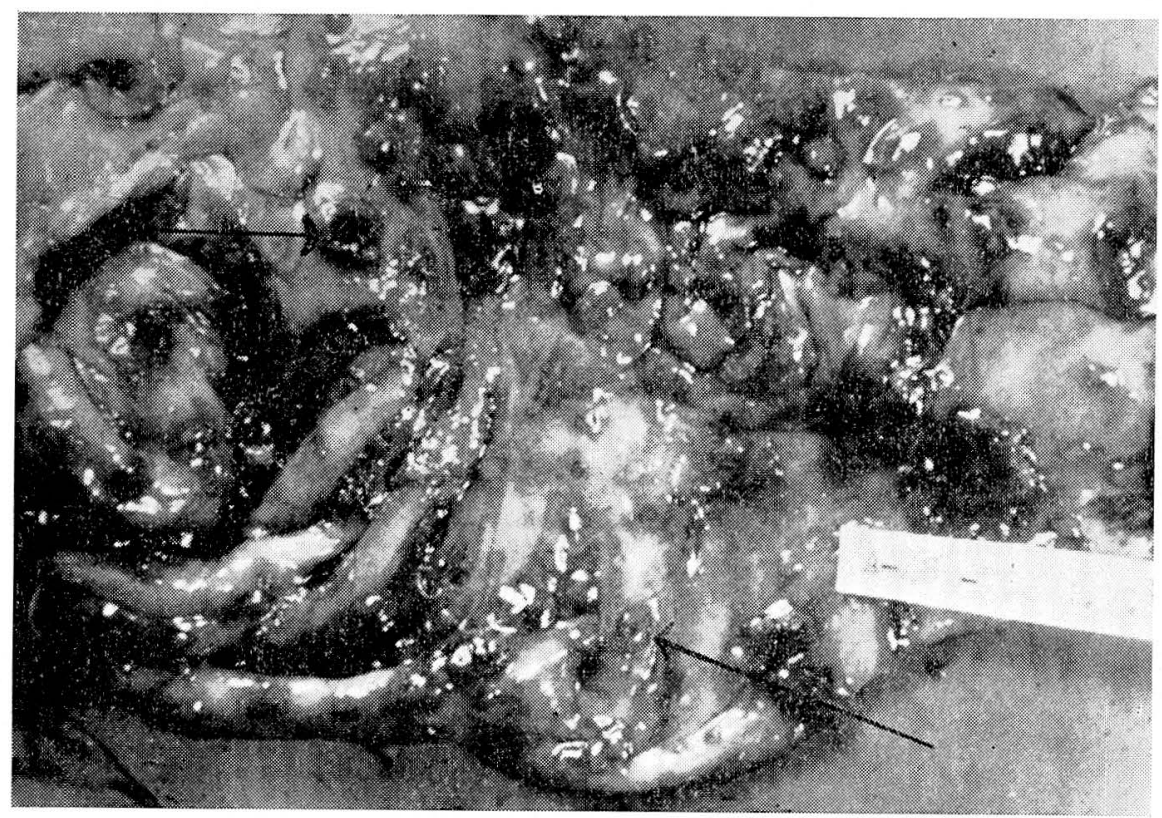

FICURA No 1 - La mucosa del colon presenta múltiples úlceras típicas de amibiasis. 
Enero-Febrero 1973

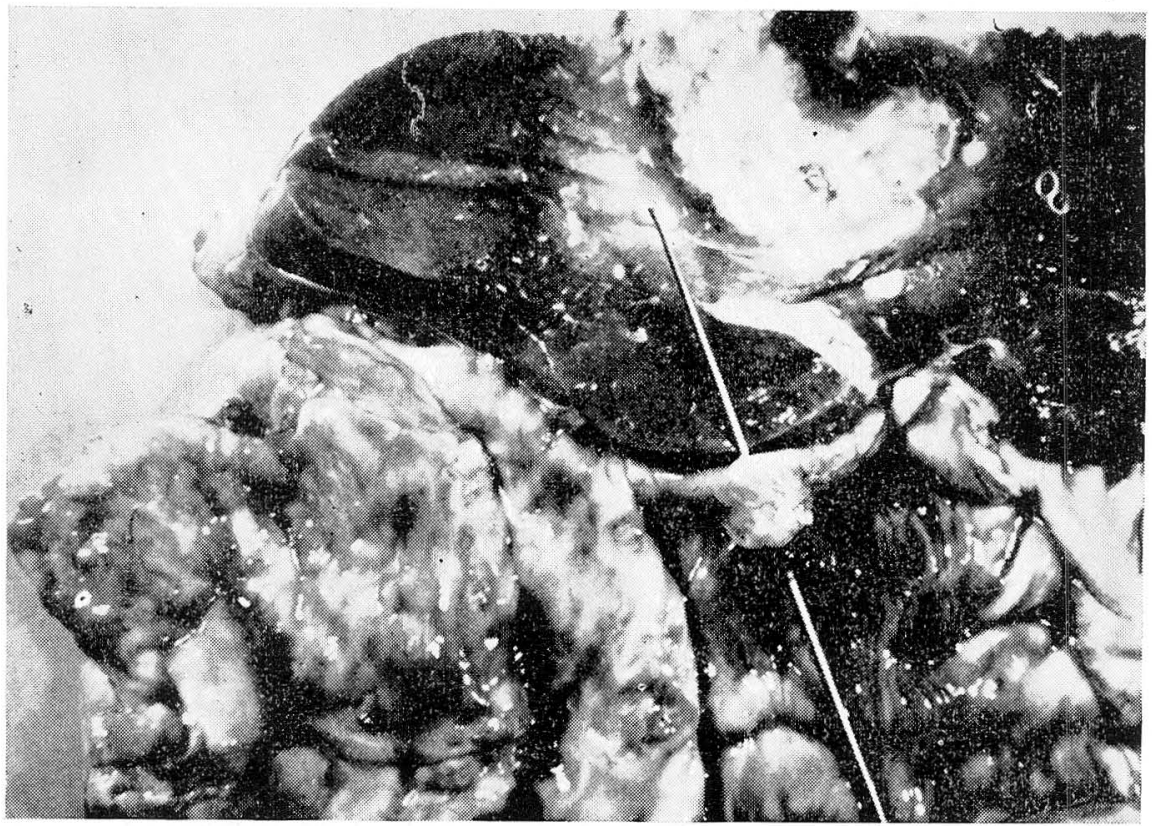

FIGURA No 2 - El estilete muestra una úlcera amibiana perforada, adherencias del colon transverso con la cara inferior del hígado, y éste con un gran absceso amibiano.

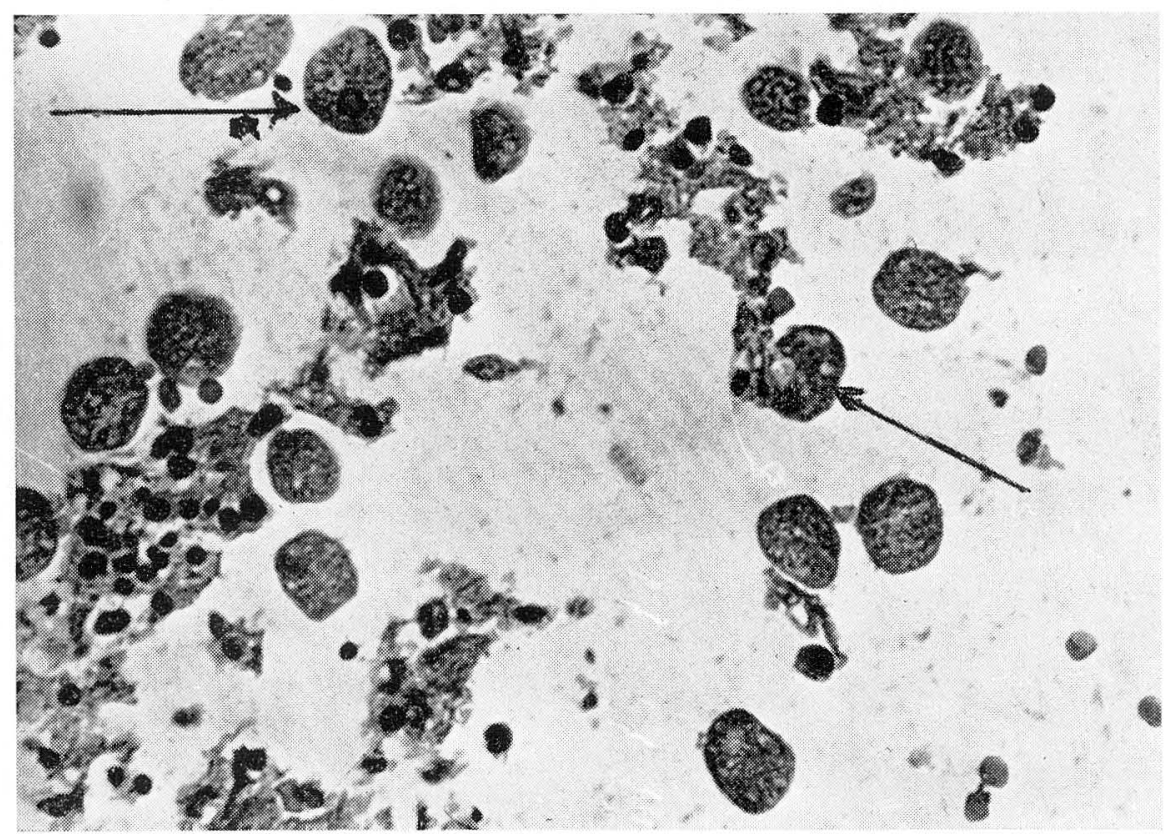

FIGURA No 3 - Trofozoircs de amibas, algunos presentan evidencia de eritrofagocitosis. 
31,5 semanas, por presentar dolor en bajo vientre y hemorragia genital desde las 9 horas del día 14 de Noviembre. Relata que desde hace 4 semanas viene presentando deposiciones diarreicas de mal olor.

Antecedentes Ginecológicos: Menarca 14 años. Ciclos $30 \times 3$.

Antecedentes Obstétricos: G5 P3 Al U.R.: III-21/64.

Examen Físico: Pésimas condiciones generales, anémica, deshidratada, edema grado I de los miembros inferiores. T.A. $9 \times 8$. Pulso 180 $x \mathrm{~m}$. Ruidos cardíacos taquicárdicos, velados. Al examen general lo único llamativo es una hepatomegalia discreta.

Examen Obstétrico: Altura uterina $25 \mathrm{cms}$.; útero hipertónico, doloroso a la palpación. TV: Vagina sana; cuello posterior, permeable a un dedo; membranas íntegras. Feto en situación transversa. Ruidos fetales negativos.

Impresión Diagnóstica :

1 Desnutrición severa.

$2^{\circ}$ Anemia severa.

3o Abruptio placentae.

40 Embarazo más o menos 30 a 31,5 semanas, con feto muerto en situación transversa.

\section{Evolución y Tratamiento:}

Es tratada con Digital, sangre total. Temp. 39,2 grados. T.A. $10 \times 6$. Eliminación urinaria escasa; vómito amarillento. Deposiciones diarreicas de aspecto sanguinolento, de mal olor. Dolor abdominal tipo "retortijón".

Al examen rectal se encuentran masas polipoides. Al examen proctológico masa a $7 \mathrm{cms}$. del reborde anal, blanda, irregular, circular, con sensación de ulceración que sangra al tacto.

I.D.: Ca. de recto, Carcinomatosis peritoneal.

Continúa deteriorando el estado general, persisten la anemia y la enterorragia, el abdomen se hace doloroso y se distiende. A las 23 horas del día 11 de Noviembre, fallece.

Los exámenes de laboratorio revelaron hipoproteinemia marcada, anemia, infección urinaria severa. Serología positiva.

Autopsia: No 184-64 (I.M.I.) :

Resumen de elsiones (definitivo).

Colitis amibiana severa.

\section{Enteritis membranosa (en yeyuno).}

Peritonitis fibrinopurulenta.

Ascitis ( 1.400 de Líq.). dad).

Hidrotórax bilateral (100 cc. en cada cavi-

Estado septicémico.

Esplenomegalia.

Desnutrición.

Degeneración grasa hepática severa.

Congestión y edema pulmonar moderados.

Pielonefritis crónica.

Parasitismo intestinal por escasos áscaris lumbricoides.

Embarazo de aprox. 30 semanas, feto muerto in útero, femenino, 1.130 grs. de altura.

En resumen se trata de una paciente desnutrida con manifestaciones de ella en corazón e hígado, complicada con enteritis bacteriana y colitis amibiana severa perforada que la lleva a una peritonitis fibrinopurulenta. La paciente muere en septicemia, debido a su estado peritoneal.

\section{SEGUNDO CASO :}

HISTORIA CLINICA No 79.642.

Edad: 39 años.

Fecha de Ingreso: VIII-3/65.

Fccha de muerte: VIII-9/65.

Causa de Ingreso: Epigastralgia, cefalalgia, poliaquiuria, náuseas, diarrea.

Enfermedad actual: Ingresa al I.M.I. por presentar epigastralgia, cefalalgia, polaquiuria, náuseas, diarrea abundante desde hace aproximadamente un mes; 18 horas antes del ingreso, no ha sentido movimientos fetales.

Antecedentes Ginecológicos: Menarca: 14 años, ciclos $30 \times 4$; U.R. Dic. 20/64.

Antecedentes Obstétricos: G5 P4. Partos normales.

Examen Físico: Regular estado general. T.A. $13 \times 10 \mathrm{P}: 100 \times \mathrm{m}$.

Examen Obstétrico: Altura uterina 28 ctms. T.V. Cuello grueso, dilatación 3 cts.; membranas íntegras. Feto único en presentación de vértice, fluídos fetales negativos. 

nas.

Edad del embarazo más o menos 32 semaEvolución y Tratamiento:

Cinco horas después de su ingreso tuvo un parto espontáneo con feto muerto, masculinc, 1.900 grs.

Continúa con diarrea. Tratamiento a base de Antidiarreicos y sueros dextrosados. Presenta ademá 's vómito, deshidratación marcada. Abdomen distendido y doloroso a la palpación; se piensa en una pelviperitonitis post-endometritis; se practica raspado uterino extrayéndose restos ovulares de mal olor y se trata con antibióticos (Penicilina, Cloranfenicol).

Continúa deteriorándose su estado general, la distensión abdominal es mayor. Fallece en estado de Shock el día 9 de Agosto a las 19 horas.

Laboratorio: Anemia marcada, infección urinaria severa. Coprológico: Giardias, amibas histolíticas: xxx. Rad. simple de abdómen: en posiciones horizontal y vertical se observan signos de pseudooclusión intestinal y reacción peritoneal.

\section{Autopsia No 105/65. I.M.I. \\ Resumen de lesiones (definitivo).}

Colitis amibiana severa: La mucosa está casi en su totalidad destruída; la superficie está cubierta por exudado necrótico y purulento; hay además edema y fibrosis de la pared con neoformación vascular. Se ven numerosas colo. nias bacterianas y grupos de trofozoitos y quistes de amiba histolítica; abscesos en la pared y reacción inflamatoria del peritoneo. El Pas deja ver numerosos quistes y trofozoitos de amibas; existen varias perforaciones del colon sigmoide formando un absceso con la pared abdominal.

Peritonitis localizada.

Ascitis (500 cc.).

Esofagitis severa.

Edema y congestión pulmonar. Hidrotórax bilateral (300 cc. en cada cavi(lod).

Bazo séptico.

\section{TERCER CASO:}

HISTORIA CLINICA No 185679.

Edad: 36 años.

Focha de Ingreso: $\times-24 / 68$.
Fecha de muerte: IX $1 / 69$

Causa de Ingreso: Dolor en hipocondrio derccho, astenia, adinamia marcada.

Enfermedad actual: Ingresa al I.M.I. en pésimas condiciones generales, astenia, adinamia marcada; dolor tipo cólico de gran intensidad localizado en hipocondrio derecho, vómito; dice haber presentado un aborto hace 3 días, nicga maniobras abortivas.

Antecedentes Ginecológicos: Menarca 14 años. Ciclos $30 \times 3$.

Antecedentes Obstétricos: G12 P9 A2. UR: no precisa.

Examen Físico: Pésimas condiciones generales. T.A. $11 \times 7$. P.: $110 \times \mathrm{m}$. Intensamente deshidratada y con tinte subictérico.

Examen Obstétrico: Cuerpo: Discretamente aumentado de tamaño. T.V. Cuello blanco, abierto, se extraen restos ovulares fétidos. Especuloscopia: Cuello congestivo, granuloso, friable.

Al examen del abdomen se encuentro moderadamente distendido, ruidos intestinales presentes. Hepatomegalia: 8 cts. por debajo del reborde costal. Se hace la impresión diagnóstica de Aborto séptico.

\section{Evolución y Tratamiento:}

La enferma empeora día a día, la deshidratación se hace más notoria a pesar del tratamiento indicado; la defensa abdominal aumenta. Previo intento de mejorar el estado general y combatir la deshidratación y con el diagnóstico de peritonitis 4 días más tarde a su fecha de ingreso se decide laparotomizar a la enferma, practicándosele una histerectomía abdominal total con salpingooforectomía izquierca y drenaje de peritonitis (cantidad aprox. 1.500 cc. de material purulento).

En el post-operatorio inmediato presenta baja tensional severa $y$ sostenida entrando en franco shock y a pesar del tratamiento con vasopresores, fallece en las primeras horas del día $1^{\circ}$ de Noviembre 1969.

Autopsia No 086-68 (I.M.I.).

Resumen de lesiones (definitivo).

Estado post-histerectomía con $\mathrm{SCl}$ y drenaje de peritonitis.

Peritonitis generalizada y severa.

Colitis amibiana perforada y abierta a cavidad. 
Abscesos hepáticos múltiples amibianos.

Edema pulmonar agudo.

\section{CUARTO CASO:}

\section{HISTORIA CLINICA No 44.328.}

Edad: 30 años.

Fecha de ingreso: VI-28/68

Fecha de muerte: VI-7/68.

Causa de Ingreso: Diarrea, dolor abdominal.

Enfermedad actual: Ingresa al I.M.I. por presentar diarrea con moco y sangre de varios días de evolución, acompañada de dolor abdominal difuso.

Antecedentes Ginecológicos: Menarca: 14 años. Ciclos $26 \times 5$.

Antecedentes Obstétricos: G7 P6. Embarazos y partos normales. U.R. Nov. 15/67. Durante el embarazo actual ha presentado episodios de diarrea en varias ocasiones.

Examen Físico: Deshidratación moderada. T.A.: $12 \times \mathrm{m}$. P: $100 \times \mathrm{m}$. Temp. $37^{\circ}$

Examen Obstétrico: Altura uterina 24 cts. T.V. Cuello corto, cerrado. Membranas íntegras, feto único, presentación de vértice, cefálica, flotante. Ruidos fetales positivos, normales.

Diagnóstico de Ingreso: G7 P6. Embarazo de más o menos 32 semanas. Diarrea amibiana.

\section{Evolución y Tratamiento:}

Se instala tratamiento a base de antidiarreicos, antiespasmódicos, hidratación y sangre. Presenta dos días más tarde parto espontáneo, sexo femenino, 1050 grs. 10/10. Continúa en pésimas condiciones generales; las deposiciones diarreicas aunque escasas son frecuentes; edema discreto de miembros inferiores. Rad. simple de abdomen: Distensión de asas delgadas. Cuadro radiológico sugestivo que corresponde a un íleo reflejo sin que pueda descartarse una obstrucción intestinal alta.

Su estado general empeora día a día. Laparotomía tres días más tarde, encontrándose pertionitis y múltiples perforaciones de colon, de origen amibiano. La intervención consiste en una colectomía total e lleostomía.

Laboratorio: Anemia marcada. Coprocultivo: Proteus vulgaris.

Autopsia No 038-68.
Estado post-colectomía total con iliostomía por amibiasis difusa severa generalizada con úlceras múltiples perforadas y peritonitis fibrinopurulenta generalizada. Ulceras múltiples amibianas en la mucosa rectal y en todo el recto. da.

Peritnoitis fibrinopurulenta severa generaliza-

Desequilibrio electrolítico.

Anemia severa.

Abscesos hpeáticos amibianos múltiples.

Hidrotórax izquierdo.

Bronquitis crónica.

Pielonefritis bilateral.

Focos bronconeumónicos en ambos campos pulmonares.

COMENTARIO: Mujer de 30 años de edad con un embarazo de más o menos 30 semanas a quien se le practica una colectomía total con lleostomía por amibiasis severa generalizada. Tiene un parto prematuro y fallece cinco días después de la intervención por peritonitis generalizada y desequilibrio hidroelectrolítico. En este caso también se pone de presente la gran virulencia de la amibiasis intestinal ocasionándole serias complicaciones que la llevaron finalmente a la muerte.

\section{QUINTO CASO:}

HISTORIA CLINICA No 336082.

Edad: 22 años.

Fecha de ingreso: VIII 12/69.

Fecha de muerte: VIII 15/69.

Causa de Ingreso: Dolor súbito de hemitórax e hipocondrio derecho, tos, fiebre, escalofrío, vómito.

Enfermedad Actual: Paciente puérpera de 12 días consultó por presentar dolor súbito en hemitórax e hipocondrio derecho, acompañado de tos con expectoración, fiebre, escalofrío y vómito bilioso, desde la iniciación del puerperio. Antecedentes de ictericia hacía un mes la cual permaneció por 8 días.

Antecedentes Ginecológicos: Menarca: 11 años. Ciclos: $28 \times 3$.

Antecedentes Obstétricos: G3 P3 AO.

Examen Físico: Malas condiciones generales. T.A.: $12 \times 7$. Temp. 35,5 P. $142 \times$ m. Respiración ruda en hemitórax izq.; disminución de 
la motilidad en H.D. con matidez y abolición del murmullo vesicular en esa zona.

Abdomen blando. Hepatomegalia 8 cts. por debajo del reborde costal.

Examen Obstétrico: Utero subinvolucionado. Cuello entreabierto a través del cual se palpan abundantes restos placentarios. Anexos: normales. Con diagnóstico de neumonía basal derecha, derrame derecho, leucocitosis, absceso subfrénico se hospitaliza. Posteriormente se hicieron los diagnósticos de Insuficiencia cardíaca congestiva, infarto pulmonar, septicemia.

Evolución y Tratamiento: Se le practica revisión uterina; presenta obnubilación marcada, hipotensión, excitación, oliguria con hematuria y melenas. Se trata con penicilina cristalina, estreptomicina, líquidos parenterales, oxígeno. Continúa en malas condiciones generales. Inconsciente, piel fría y sudorosa, hipotensión. El día 15 de Agosto/69 hace paro respiratorio y fallece.

Laboratorio: leucocitosis marcada, anemia severa.

Autopsia № 273/69:

Resumen de lesiones: (definitivo).

Colitis ulcerativa amibiana.

Absceso hepático amibiano con perforación a hemidiafragma derecho y formación de piotórax ( 2.500 cc.).

Colapso pulmonar derecho severo.

Congestión aguda hepática.

Gastritis congestiva.

Utero con restos placentarios y ligera endometritis.

\section{SEXTO CASO :}

HISTORIA CLINICA No 246157.

Edad: 16 años.

Fecha de ingreso: $\mathrm{V}-4 / 72$.

Fecha de muerte: $V-14 / 72$.

Causa de Ingreso: Hemorragia genital escasa. Dolor tipo cólico, diarrea, fiebre.

Enfermedad actual: hemorragia genital escasa, fiebre, diarrea; disuria desde hace varios días. Antecedentes de hemorragias rectales hace dos meses.

Antecedentes Ginecológicos: Menarca: 14 años, ciclos $30 \times 3$. U.R. XI-11 de 1972 .
Antecedentes Obstétricos: G1 PO.

Examen Físico: Deshidratada. T.A.: $8 \times 6$ $P: 100 \vee \mathrm{m}$.

Examen Obstétrico: Altura uterina: $24 \mathrm{cts}$. T.V.: Cuello blando largo, permeable 1 dedo; membranas íntegras. Feto único, presentación cefálica, vértice, ruidos positivos normales. Se hace la impresión diagnóstica de amenaza de parto prematuro.

Evolución y Tratamiento:

Continúa con fiebre, diarrea, disuria. Tres días más tarde presenta parto espontáneo con feto femenino, 800 grs. 1/10. Continúan las deposiciones diarreicas. Una radiografía de abdomen simple revela una marcada dilatación de asas delgadas con ausencia de gas en el recto. Compatible con reflejo. Deshidratada. Se hace el diagnóstico de sepsis. Shock séptico. Se prescribe Prostafilina, Kantrex, Penicilina. Hidratación. Empeora su estado general y la enferma fallece el $V-14 / 72$ a las $8: 50$.

Los exámenes de laboratorio mostraron anemia, desequilibrio hidroelectrolítico marcado.

Autopsia No A-066/72.

Resumen de lesiones (definitivo).

Colitis amibiana severa (úlceras de $3 \times 2$. $\mathrm{mm}$. de diámetro).

Desequilibrio electrolítico.

Peritonitis crónica localizada en colon ascendente.

Edema y congestión pulmonar.

Utero de post-parto normal.

Pielonefritis crónica focal.

Bronconeumonía bilateral moderada.

Anemia aguda.

\section{Resumen y Conclusiones}

1. Se presenta la frecuencia de la amibiasis intestinal en varios sitios del mundo, con menor incidencia en Río de Janeiro ( $3 \%$ ) y mayor en el Congo $(84 \%)$ se le dá importancia a los factores socio-económico, ambiental, higiénico.

2: También se presenta la frecuencia de la amibiasis intestinal en di- 
versas regiones de nuestro país, frecuencia que varía entre el $20,1 \%$ y el $66 \%$.

3! Se comenta como los trabajos sobre tal parasitosis asociada al embarazo son muy escasos. Según Pérez $y$ otros esta modalidad es del 52,9\%.

4․ Esta asociación en la Consulta Externa de la Clínica San Pedro Claver de Bogotá es del 26\%; en el Centro № 9 del Distrito Especial del $34 \%$ y en el Instituto Materno Infantil de Bogotá, del 38\%. Se hace hincapié que estas cifras podrían ser más altas si se tiene en cuenta que para su investigación solamente se ordenó un examen coprológico y no otros procedimientos esenciales para su búsqueda.

5: Los diferentes síntomas clínicos y signos que suelen presentarse en las formas agudas y crónicas de la disentería amibiana, son comentadas.

6: Se transcriben los conceptos de algunos autores sobre la influencia que la amibiasis intestinal ejerce sobre el feto, haciéndose hincapié en la forma aguda, casi siempre es fatal para él.

7: Parece que el estado grávidopuerperal puede ofrecer condiciones propicias para aquellas lesiones amibianas latentes, desarrollando una reactividad muy marcada. Según algunos autores, el embarazo aumenta la gravedad de la infección; según otros, basados en experimentos en animales de laboratorio, los corticoides y la progesterona que se producen durante la gravidez agravan la infección amibiana ocasionando complicaciones serias como perforaciones intestinales, abscesos hepáticos y pulmonares, peritonitis, etc.

\section{Sumario}

Se presentan y comentan 6 casos de amibiasis intestinal asociada con embarazo, demostrando claramente la dificultad para hacer un diagnóstico previo, la exaltación de la virulencia de tal parasitosis, la producción de complicaciones graves (perforaciones intestinales, peritonitis, abscesos hepáticos y pulmonares, colitis severas, etc. todas ellas capaces de llevar las enfermas a la muerte.

Se aconseja pensar en tal asociación en embarazadas con diarrea, especialmente si presentan pujo, tenesmo, dolor abdominal, fiebre y vómito.

\section{Summary}

Six cases of intestinal amebiasis associated with pregnancy are presented and discussed, clearly proving the difficulty of accomplishing a previous diagnosis, the increase in the virulence of said parasitosis, the appearance of serious complications (intestinal perforations, peritonitis, hepatic and lung abscesses, severe colitis, etc.), all capable of leading the patients to death.

It is recommended that thought be give to such association in pregnant women suffering diarrhea, particularly when tenesmus, abdominal pain, fever and vomitus are present.

\section{BIBLIOGRAFIA}

1 ROVINSKY J. GUTTMACHER A. F. Complicaciones Médicas, Quirúrgicas y Ginecológicas en el embarazo. Editorial Suramericana. 1967.

2 ESCORCIA DE EVA. Amibiasis und $X X$ ihre Komplikationen. Tesis de grado. Leipzig 1967.

3 ROJAS W. LEIDERMAN E. VELEZ H.: Tratamiento de la amibiasis intestinal con clorofenoxamida. Ant. Med. 13: 529, 1963. 
4 KOFOID C. A.; SWESY O.; BAYERS S. M. : $A$ report and investigations of intestinal protozous infection at Sta. Marta, Colombia in the Hospital of the United Fruit Co. With special reference to the incidence of amaebiasis. United Fruit Company Med. Dept. 14th Anual Report 140-153, 1926.

5 HERNANDEZ C. R. Nuevo tratamiento de la amibiasis intestinal. Rev. Fac. Med. Bogotá. 17: 415-455, 1949.

6 RESTREPO A.: Contribución al estudio de las parasitosis en el Valle de Medellín. Boletín Clínico Año VI (1-2): 1-56. Nov.-Dic. 1939.

7 DOMINGUEZ R.: Amibiasis Infantil y Amibiasis de Lactantes. Rev. Col. Ped. Puericultura. Año X (1) 5-47. 1950.

8 BOTERO LOPEZ: Amibiasis y Parasitosis Intestinal en el Hospital Mental de Antioquia. Anat. Med. Oct. No 5, 1958.

9 FAUST, E. C.: Parasitologic survey in Cali, Departamento del Valle, Colombia. Am. J. Trop. Med. \& Hyg. 7: 4-15, 1958.

10 DUQUE O.; ARCILA G.; ZULUAGA H.: Estudio comparativo de la infección por Entamosiba histolítica y otros parásitos intestinales en indios y blancos de Chocó (Colombia). Ant. Med. (9): 365-385, 1959.

11 RESTREPO M. Estudio parasitológico de una región del Amazonas Colombiano. Ant. Med. 12 (8) : 462-484. Sept. 1962.

12 ZULUAGA HORACIO: Estudio epidemiológico sobre amibiasis. Comparación de una comunidad de Puerto Rico con una de Colombia. Anat. Med. Vol. 15 No 5, 1965.
13 DUQUE OSCAR. Amibiasis Fatal en Colombia. Estudio anatomo-clínico de 176 casos. Ant Med. 18: 10, 1968.

14 LEON JUAN. Tratado de Obstetricia. Ediciones M. Segura. 1963.

15 PEREZ M. L. Compendio de Clínica Obstétrica. López Libreros Editores S. R. L. 1962.

16 CLIFFORD L. y cols. Parasitosis y embarazo. Complicaciones médicas, quirúrgicas y ginecológicas en el embarazo. Editorial |r| teramericana, 1967.

17 MITRA S.: Tropical diseases and pregnancy. En British Obstetrical and Gynecological Practice. 2a. ed. Capítulo 15 editado por E. Howlland y O. Bourne, W. Heineman, London, 1959.

18 TEODOROVIS, S.; INGALLS, J. W. Y GREENBERG L.: Effects of corticosteroids on experimental amebiasis. Natura, 197: 86, 1963.

19 BIAGI F.; ROBLEDO, E.; SERVIN H. y MARVAN G.: Influence of some steroids in the experimental production of amebic hepatic abscess. Am. J. Trop. Med. 12: 318, 1963.

20 SANTOYO, E.; VILLALOBOS M. E. y VASQUEZ, A. M.: Consideraciones acerca de 100 casos de abscesos hepáticos. Rev. Med. Hosp. General (Mex), 23; 469-482, 1960.

21 RIVERA H. H.: Peritonitis amibiana en el estado grávido puerperal. Rev. Med. Hosp. General (Mex) 23; 773-778, 1960.

22 MODY U. R.: Corticosteroids in laten amoebiasis. Brit. Med. J. 2: 1399 (correspondence), 1959.

23 VILLAREJOS V. M.: Cortisone and experimental amebiasis in the eat. J. Parasitol. 48: 194, 1962. 